\title{
Partial reinforcement with a small number of trials: Two acquisition trials'
}

GARVIN MCCAIN AND ERIN RAYFIELD BROWN

ARLINGTON STATE COLLEGE OF THE UNIVERSITY OF TEXAS

Two groups of rats were run in a straight alley. One group (NR) was given one nonreinforced trial and one reinforced trial. Another group was given two reinforced trials. Based on goal times, Group NR was more resistant to extinction. This finding supports previous work using a small number of acquisition trials.

As reported in McCain (1966, Experiment 2) a minimum number of two acqusition trials, with a NR reinforcement pattern, resulted in greater resistance to extinction than a RR pattern. The present study employed the same reinforcement pattern with some alterations in procedure.

The original procedure was devised to test assumptions of the frustration hypothesis concerning the number of trials necessary to produce the PRE -"... the PRE will be evident only after some critical number of training trials..." (Amsel, 1958). Presumably the mediating reaction $r_{f}$ and its stimulus consequences, the crucial mechanism for the frustration interpretation of PRE (Amsel, 1958), becomes conditioned to the instrumental response only after a more extensive training period than two trials.

McCain (1966, Experiment 2) used $60 \mathrm{sec}$. access to wet mash as the reinforcer. It is possible that this period resulted in some satiation for the $\mathbf{R R}$ group. In the present study, along with changing from a period of access to food to a fixed amount of food, the magnitude of the reinforcer was substantially reduced.

\section{Method}

Subjects. Subjects were 66 albino rats of the Wistar strain from the Arlington State College colony. The Ss were approximately 90 days old at the beginning of the experiment and approximately equal numbers of each sex were used.

Apparatus. The apparatus was a straight alley arranged so that start, run and goal times could be measured. A more complete description may be found in McCain \& Power (1966).

Procedure. The habituation and experimental procedures for this experiment were the same as in Experiment 2 (McCain, 1966) except for these differences: Exploration trials were given in the same apparatus in which the animals were to be tested, experimental trials were begun on Day 11, and the ITI was $15 \mathrm{sec}$. An important difference was in goal box (GB) confinement time. Reinforcement for Ss was four $97-\mathrm{mg}$ Noyes pellets and Ss were removed from GB immediately after eating (in previous experiment Ss ate wet mash for $60 \mathrm{sec}$.). Also, on nonreinforced acquisition trials and extinction trials Ss remained in the GB for only $15 \mathrm{sec}$. (as opposed to $30 \mathrm{sec}$. in the previous experiment).

Any $S$ that failed to reach the GB within $30 \mathrm{sec}$. on first acquisition trial was dropped from the study immediately. This same 30-sec. criterion was applied to the first extinction trial. A total of six Ss were dropped, three from each group, for exceeding the criterion, failure to eat, or experimental error.

The number of acquisition trials (two) and extinction trials (seven) were the same as in the earlier experiment. The partial group (NR) received a NR reinforcement pattern and the consistent group (RR) a RR pattern. Ss from the groups were run in an ABBA order.

The first extinction trial is not included in the extinction data because the Ss in the consistent group had not experienced nonreinforcement before this trial and, therefore, it cannot realistically be called an extinction trial. A constant of +1 was added to the medians from the remaining two blocks of three extinction trials. This score was transformed to a logarithm.

Results

In Fig. 1, the transformed start, run and goal times are shown for individual acquisition trials (A), the first extinction trial $\left(E^{1}\right)$ and blocks of three E-trials. As may be noted the groups are very close and there are not significant differences on $A^{1}$. Group NR had longer times on all measures of $A^{2}$. The differences are significant for start $(p<.02)$ and goal times $(p<$ $.04)$, and approach the usually accepted level $(p<.10$ $>.05$ ) for run times. There are no significant differences in $E^{1}$ times. The largest apparent difference is in run times and this is not substantial $(p>.25)$. In brief, there is little difference between groups in

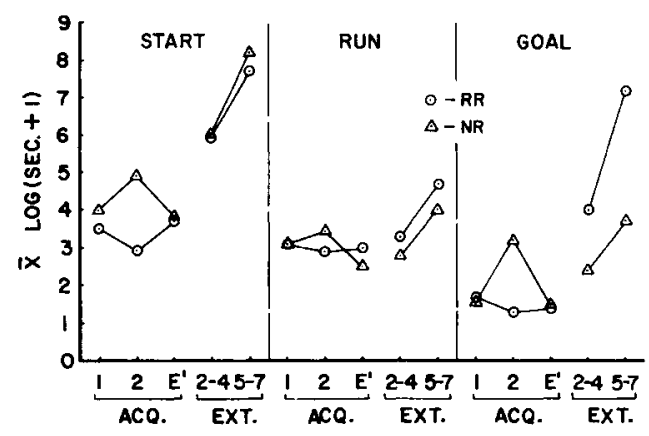

Fig. 1. Individual acquisition trials and blocks of extinction trials for start, run and goal times. 
acquisition except on $\mathrm{A}^{2}$; here Group $\mathrm{RR}$ performed more vigorously.

As seen in Fig. 1, there is very little difference in extinction in the start or run measures. The difference in goal times appears substantial. The comparisons across measures seem to reflect an increasing difference between groups as the goal area is approached. A Lindquist Type VI analysis was applied to the transformed extinction data. The overall Groups effect is not significant $(F=2.33, d f=1 / 58, p>.10)$. The Groups by Measures interaction is significant $(F=4.94, \mathrm{df}=2 / 116, \mathrm{p}<.01)$. The difference between goal times is significant $(t=2.63, \mathrm{df}=58, \mathrm{p}<.015)$ with Group NR running faster in this section. None of the other group comparisons reached the .05 level.

\section{Discussion}

In the earlier two-trial study (McCain, 1966, Experiment 2) a straight alley with 38-in. runway was used and the running time was taken over the entire length. In the present experiment a 48-in. alley was used, with measures taken in short segments near the start box, in mid-alley, and near the goal. The fact that Group NR ran faster in the goal area during extinction supports the findings of earlier studies that the PRE, or some very similar effect, can be produced with the very minimum number of acquisition trials.

This is the first study from this laboratory using a small number of A-trials in which goal times have been measured separately. A study from another laboratory (Spence, Platt, \& Matsumoto, 1965) reported three measures similar to those used here. Spence found their measures to be roughly comparable, "Although the results for the three measures differed somewhat they showed more or less the same trends." If there is other than chance difference between the present results and Spence's, it may well be related to the stage of acquisition represented. For the moment one could speculate that the effects of acquisition would be seen first in the goal a rea, spreading rapidly to other parts of the runway as training progresses. Because of the rather limited magnitude of rein- forcement available, satiation does not seem to be a critical variable in this situation. In another study (McCain, 1966, Experiment 3) reward magnitudes of roughly the same order used in this study were arranged for the partial and consistent reinforcement groups in order that they would receive the same total amount of food. That study, with four acquisition trials, achieved results quite consistent with the present findings.

The previous two-trial study (McCain, 1966, Experiment 2) was interpreted in terms of the modified aftereffects or sequential hypothesis (Capaldi, 1966). At present there does not seem to be any reason to change this analysis.

As indicated in earlier studies (McCain, Love, \& Gruer, 1962; McCain, 1966), the early development of the PRE seems to argue against those assumptions in the frustration hypothesis leading to the conclusion that an extended period of training is necessary to produce the PRE. The evidence to date does not rule out the possibility that frustration plays a part in some stage of the partial reinforcement situation, but it does indicate that the early stages of training do not correspond to the processes described in the frustration hypothesis.

\section{References}

Amsel, A. The role of frustrative nonreward in noncontinuous reward situations. Psychol. Bull, 1958, 55, 102-119.

Capaldi, E.J. Partial reinforcement: A hypothes is of sequential effects. Fsychol. Rev., 1966, 73, 459-477.

McCain, G. Partial reinforcement effects following a small number of acquisition trials. Psychon. Monogr. Suppl., 1966, 1, 251-270.

McCain, G., Love, Nancey, \& Gruer, W. Extinction as a function of a small number of partially reinforced trials. Psychol. Rep., $1962,11,451-454$.

McCain, G., \& Power, R. Extinction as a function of reinforcement conditions in the start box. Psychon. Sci., 1966, 5, 193-194.

Spence, K. W., Platt, J. R., \& Matsumoto, R. Intertrial reinforcement effect as a function of number of training trials. Psychon. Sci., 1965, 3, 205-206.

\section{Note}

1. This work was supported by NIMH grant MH 12325-01. Thanks are due to Jacquelyn Moe who served as experimenter. 